\title{
Pharmacological Management of Pattern Hair Loss
}

\author{
Sandeep Suresh Sattur ${ }^{1}$ Indu Sandeep Sattur ${ }^{1}$ \\ ${ }^{1}$ HAIRREVIVE- Centre for Hair Restoration \& Skin Rejuvenation, \\ Santacruz West, Mumbai, Maharashtra, India \\ Indian J Plast Surg 2021;54:422-434.
}

\begin{abstract}
Address for correspondence Sandeep Suresh Sattur, MCh, HAIRREVIVE- Centre for Hair Restoration \& Skin Rejuvenation, 103, Options Commercial Centre, Dr Vasant Awsare Marg, Milan Subway road, Santacruz West, Mumbai, Maharashtra, 400054, India (e-mail: drsattur@yahoo.com).
\end{abstract}
Abstract
Keywords
- Pattern Hair Loss
- Minoxidil
- Finasteride
- Post finasteride syndrome
- Dutasteride
- Topical finasteride

Pattern hair loss (PHL) is a condition that worsens with time and the only way it can be slowed down is with pharmacological intervention. Pharmacological treatments for $\mathrm{PHL}$, from an evidenced-based perspective with respect to safety and efficacy, are limited to only two drugs, minoxidil and finasteride. However, there are a host of drugs being used, off-label with limited evidence. This article attempts to review the literature on this topic, and the authors add to this, with their experience of over two decades on incorporating pharmacologic treatments along with hair transplantation in their management of PHL.

\section{Introduction}

Pattern hair loss (PHL) is the most common form of hair loss seen in both genders (male pattern hair loss [MPHL] and female pattern hair loss [FPHL]). Although not a true medical disease or a threat to life, it is a cosmetic problem which technically starts at puberty and progresses with age and significantly impacts the quality of life in affected individuals. ${ }^{1-4}$ Man has tried to treat hair loss for centuries without a rational approach. ${ }^{5-7}$ Today, with internet savvy patients, most would have tried a host of remedies, online advocated treatments, over-the-counter (OTC) products, etc. With so many options available, the irony of pharmacological interventions is that to date there are only two approved drugs for PHL, both of which were not born out of a rational drug design but out of serendipity. ${ }^{3,8}$ Misinformation and resistance to open-ended treatment is the limiting factor in the pharmacological management of PHL.

\section{Role of Pharmacologic Intervention in PHL}

According to the authors, PHL has the following two components:

published online

December 13, 2021
DOI https://doi.org/ $10.1055 / \mathrm{s}-0041-1739254$ ISSN $0970-0358$.
1. The existing deficit in facial aesthetics caused by hair loss (static component), which is usually addressed by hair transplantation.

2. Potential for hair loss to get worse with time (dynamic component), which is pharmacologically managed.

Both components need to be dealt with to achieve satisfactory outcome. Ensuring that the patient comprehends this along with the fact that medical management is long-term, possibly life long, is the key to successful management. Pharmacological management is necessary for all patients of PHL due to its progressive nature and that includes patients undergoing hair transplantation. . $^{1,3,4,9,10}$

The hallmark of PHL is gradual reduction in hair shaft diameter, caused by reduced dermal papilla (DP) volume with reduced dermal papilla cells (DPCs). ${ }^{11}$ The objective of pharmacological treatment in PHL is to reverse miniaturization to improve scalp coverage and prevent or slow further hair thinning and loss.

Currently, the pharmacological options with the best level of evidence from the efficacy and safety perspectives are topical minoxidil and oral finasteride for MPHL and topical minoxidil 1 for FPHL. ${ }^{1-4}$

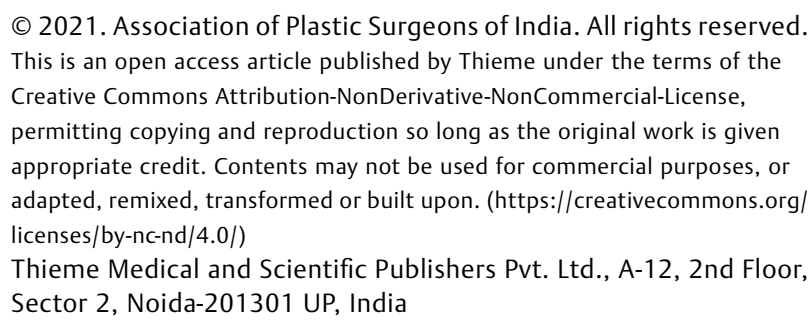


Table 1 Prior to starting treatment, it is essential to get a thorough blood workup mentioned in ${ }^{1}$

\begin{tabular}{|l|}
\hline Tests to be done before medical treatment of PHL \\
\hline Complete blood cell count \\
\hline Comprehensive metabolic panel \\
\hline TSH \\
\hline $\begin{array}{l}\text { Iron evaluation (serum ferritin, serum iron, total iron-binding } \\
\text { capacity) }\end{array}$ \\
\hline Free and/or total testosterone and DHT \\
\hline Vitamins D and B12 \\
\hline Additionally, in women-DHEAS and serum prolactin \\
\hline
\end{tabular}

Abbreviations: DHEAS, dehydroepiandrosterone sulfate; DHT, dihydrotestosterone; TSH, thyroid-stimulating hormone.

The cause of PHL is thought to be multifactorial, of which some of the elements are identified (androgens and genes) and some still under speculation (microscopic follicular inflammation, oxidative stress, prostaglandins, retinoid receptors and PPAR pathways, etc.), leaving a wide scope of therapeutic intervention. But, to date, only two molecules have been found to be helpful and safe. ${ }^{1-4,11-13}$

\section{Pretreatment Assessment}

A confirmed diagnosis made on the basis of a positive family history, hair loss which is patterned (hair loss in the frontal, midscalp and crown/vertex regions with sparing of occipitoparietal region), and presence of anisotrichosis on trichoscopy are mandatory before starting treatment (- Table 1). ${ }^{14}$

\section{Hurdles in Initiating and Maintaining Patients on Pharmacological Treatment}

The age group of patients consulting for hair loss varies between late teens to those in their sixties or seventies, with the majority of them being in their third or fourth decade of life. ${ }^{15,16}$ The younger patients are significantly distressed with the hair loss, leading to a negative body image. Moreover, most patients come armed with online information (or misinformation) and bias. ${ }^{17,18}$ It is one of the challenges faced by doctors to constantly counter this information and convince the patient of the right treatment protocols, albeit without any conflict of interest. To take oral or topical medications, which are not curative for the rest of their lives, is the most important limiting factor in initiating medical treatment. Patient compliance is key to success of pharmacological management of PHL. Regular periodic (every 2 or 3 months) photographic documentation of the hair loss status helps patients to objectively see the improvement in the hair loss status, which spurs them to persevere to the treatment protocol. Compliance to treatment is also facilitated by empathetic and patient counselling.

\section{Pharmacological Management of Pattern Hair Loss}

The approved drugs with labeled use in PHL, which have level 1 evidence for efficacy and safety with level A recommendation $^{1,4,19,20}$ are as follows:

- Topical minoxidil 2\% in MPHL and FPHL and 5\% twice a day in MPHL and once a day in FPHL.

- Oral finasteride $1 \mathrm{mg}$ in MPHL.

Off-label therapies and those with lower levels of evidence and recommendations are as follows:

- Dutasteride.

- Topical finasteride.

- Spironolactone.

- Cyproterone acetate.

- Peptides.

- Bimatoprost and latanoprost.

\section{Minoxidil}

Originally, minoxidil was an antihypertensive agent that was used largely for patients with severe and refractory hypertension. The credit for identifying its role in restoring hair goes to dermatologist Guinter Kahn who was the first to connect increased hair growth as a side effect of minoxidil usage in $1971 .^{21}$ Topical use for PHL was formally approved in 1986 (2\% for MPHL), 1993 (5\% for MPHL and 2\% for FPHL), in 2006 ( $5 \%$ foam for MPHL), and in 2014 (5\% foam for FPHL). ${ }^{22,23}$

\section{How Does Minoxidil Work in PHL}

PHL in men and women are the only labeled indications for topical minoxidil. Minoxidil is a piperidino-pyrimidine derivative (2,4-diamino-6 piperidino-pyrimidine-3-oxide) which works like a prodrug, needing conversion to its active metabolite-minoxidil sulfate (MS)-to exert its pharmacological actions. The active metabolite is responsible for vascular as well as the follicular actions of minoxidil. ${ }^{22,24}$ MS exerts its antihypertensive effect by opening of plasma membrane adenosine triphosphate (ATP)-sensitive potassium channels (KATP channels). ${ }^{22}$ The exact mechanism by which minoxidil promotes hair growth is uncertain and has been under investigation for the last few decades. Although increase in local blood flow has been suggested as a possible mechanism of hair growth, in vitro studies involving follicles in culture media have shown that minoxidil does indeed work on the KATP channels present in the hair follicle, even in the absence of any local vasculature. ${ }^{25,26}$ Various operating mechanisms contributing to the follicular benefits of minoxidil have been put forward. Apart from the effects on KATP channels, minoxidil causes upregulation of vascular endothelial growth factor (VEGF), which may be responsible for local angiogenesis, increased local growth factors-VEGF, hepatocyte growth factor (HGF) and insulin-like growth fact (IGF1), increased prostaglandin E2 (PGE2) production in dermal papilla fibroblasts by stimulation of prostaglandin 


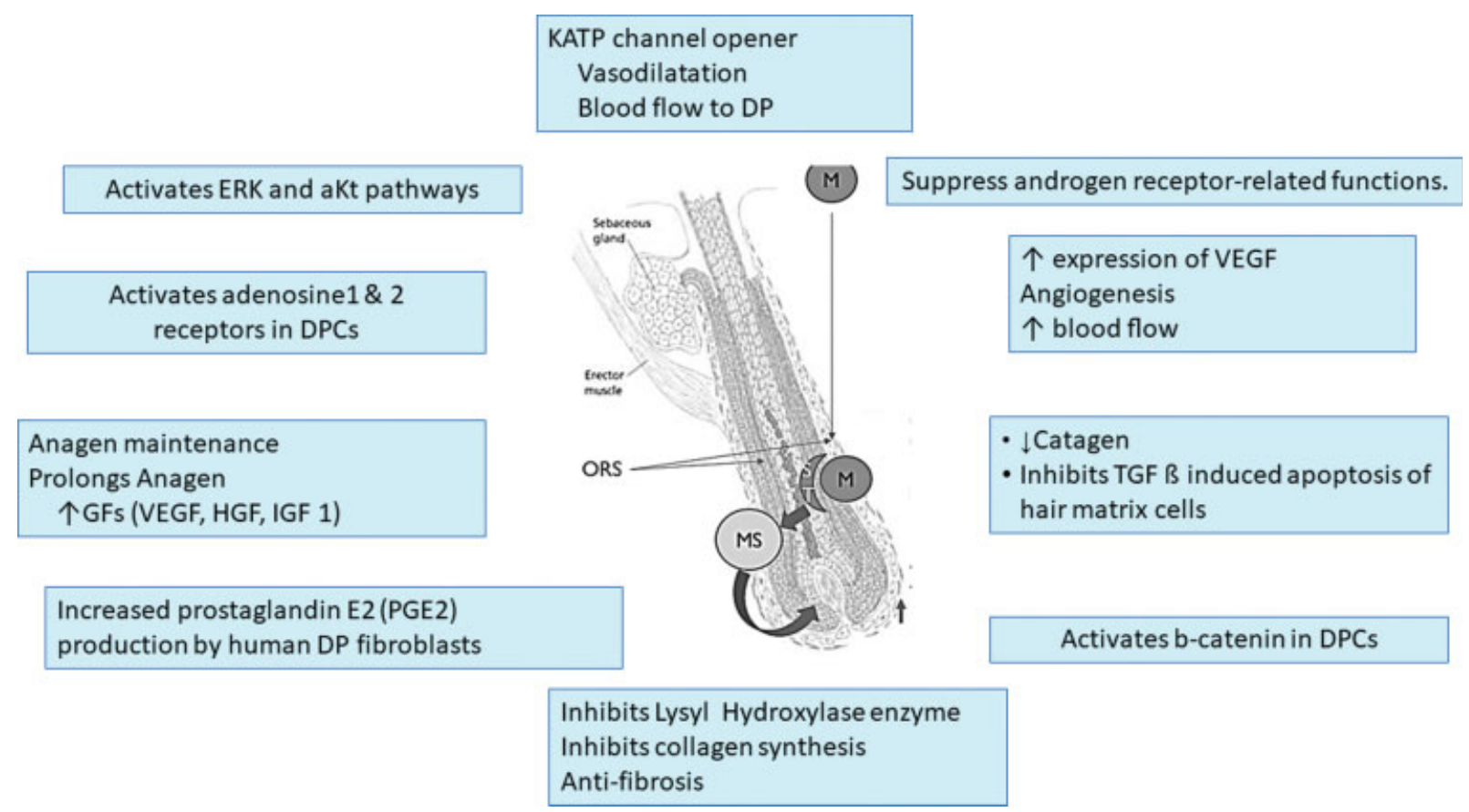

Fig. 1 Proposed mechanisms of action of minoxidil on hair follicles.

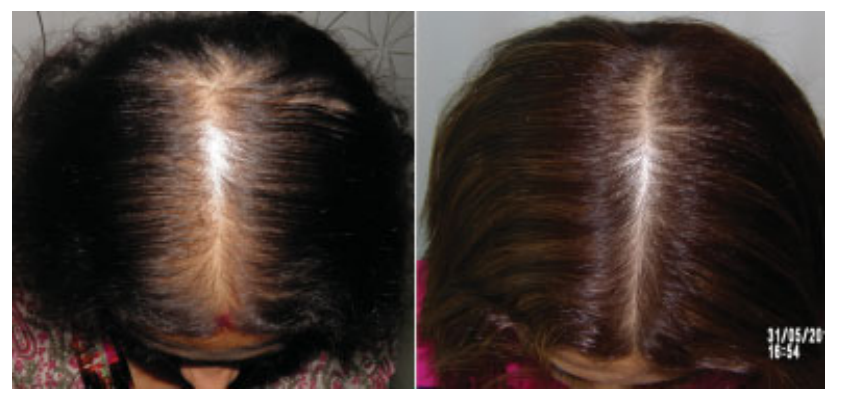

Fig. 2 Results of minoxidil in pattern hair loss (PHL).

endoperoxide synthase, upregulating the b catenin pathway, decreased catagen by inhibiting TGF b-induced apoptosis of hair matrix cells, activating ERK and aKt pathways as well as activating adenosine 1 and 2 receptors in DPCs; finally, it may also play a role suppressing androgen receptor (AR)-related functions (-Fig. 1).22,24-28

Application of minoxidil causes anagen prolongation, delays catagen progression, and shortens telogen. ${ }^{22,26,29}$

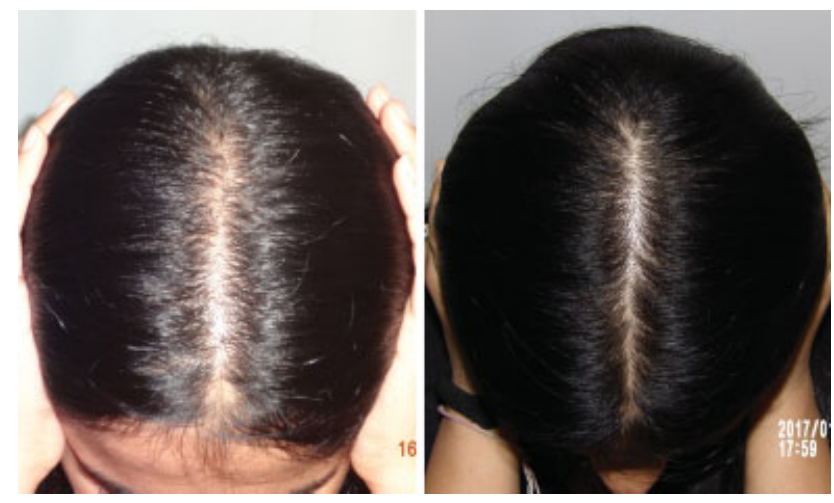

Fig. 3 Results of minoxidil in pattern hair loss (PHL).

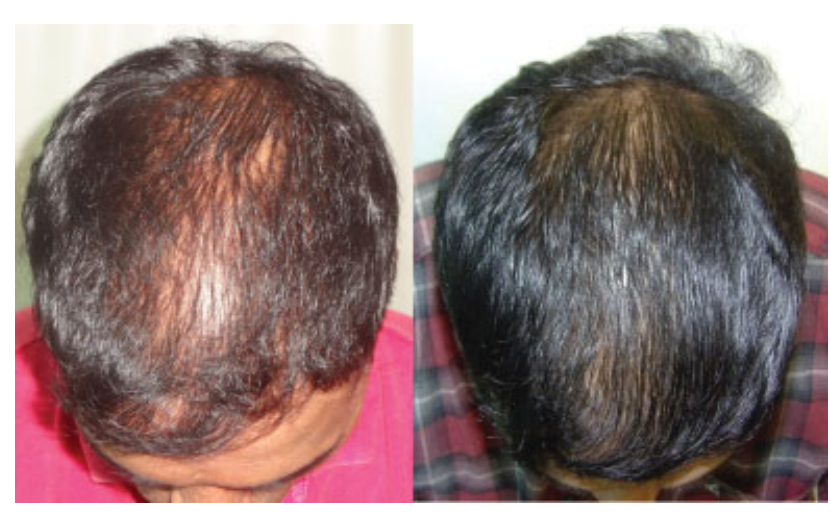

Fig. 4 Results of minoxidil in pattern hair loss (PHL).

All these actions translate into improved hair growth. Shortening of telogen results in increased shedding after initiating treatment. This is seen within a week of starting minoxidil and may last a few weeks. The benefits are objectively noticeable between 6 to 12 months after starting therapy (-Figs. 2, 3, 4).

\section{Pharmacokinetics of Topical Minoxidil}

Direct follicular action without systemic effects justifies topical use of minoxidil ${ }^{30}$ but hurdles of transdermal absorption need it to be mixed with water, ethanol and propylene glycol (PG). ${ }^{26} \mathrm{PG}$ is necessary to improve absorption but is also the cause for irritation of the skin. Limited systemic absorption (less than 99\%) limits the possibility of vascular effects. ${ }^{31}$ Minoxidil action on the follicle is dependent on the expression of sulfotransferase enzyme in the hair follicles, which can vary in the population, and this is the reason for differential effects of minoxidil. ${ }^{32}$ The levels of this enzyme can be impacted favorably (retinoic acid) or unfavorably by certain substances (aspirin, salicylates). ${ }^{32,33}$ 


\section{Adverse Effects with Topical Minoxidil}

Topical minoxidil is a safe drug which is evident from the fact that it is an OTC medication in many countries. But some minor adverse effects may be seen in some patients. These could be temporary and gradually reduce with time but sometimes the irritation is more severe, mandating discontinuation or modification of therapy.

The most common adverse effect which is also responsible for patients defaulting on treatment is PG-induced irritant dermatitis (contact allergy to minoxidil is rare). This can be significant in a subset of patients, mandating discontinuation of the treatment or change of formulation. The irritation is directly proportional to the minoxidil concentration $(5 \%>2 \%)$ - Fig. $\mathbf{5}^{26}$

Another reason why patients discontinue minoxidil is the initial increased hair fall caused due to shortening of telogen phase, and this should be mentioned to patients while starting minoxidil therapy, but the authors feel that this could be a pointer to its effectiveness in that patient.

Other adverse effects that may be seen are hypertrichosis, especially on the face in women. This is linked to the concentration of minoxidil $(5 \%>2 \%)$ or could be due to improper application. ${ }^{26}$ It is always a good idea to photograph the face with the side burn and chin regions before starting treatment, so as to document any increase in facial hair with minoxidil usage - Fig. 6 .

Many patients are reluctant to use it in work or social situations, as the hair sometimes appears to be dry, dull, and stiff. This could probably be due to improper application (delivering most minoxidil onto the hair instead of the scalp), leaving precipitate of minoxidil on the hair shaft ( - Fig. 7). This reluctance can be overcome by educating the patient on correct application techniques and use of conditioners and leaving on serums applied between minoxidil applications (-Fig. 8a and b). ${ }^{34}$

It is extremely rare for patients to complain about hypotension, but some patients may complain about palpitations, irritation or burning of eyes, weight gain, etc. ${ }^{35}$ Although it qualifies as an OTC product, topical minoxidil can have an impact on the cardiovascular system. ${ }^{35}$ This may become significant in patients with cardiac compromise. Cardiologists'/physicians' opinion regarding safety of starting topical minoxidil in such patients is important. Although not com-

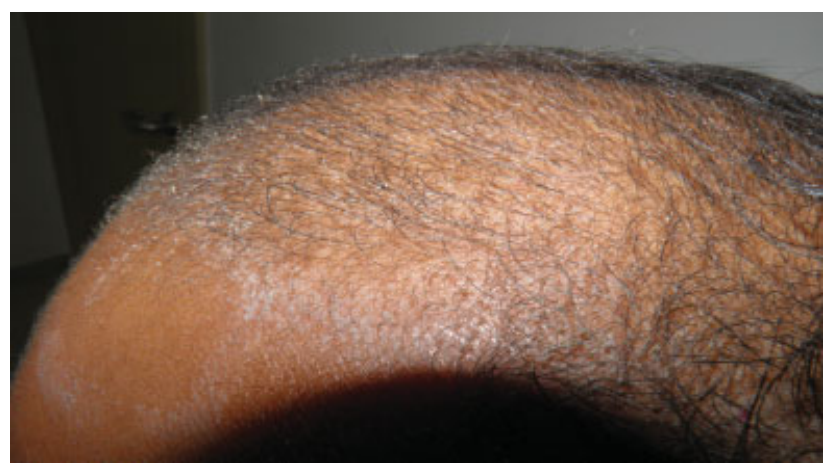

Fig. 5 Irritant dermatitis caused by minoxidil application.

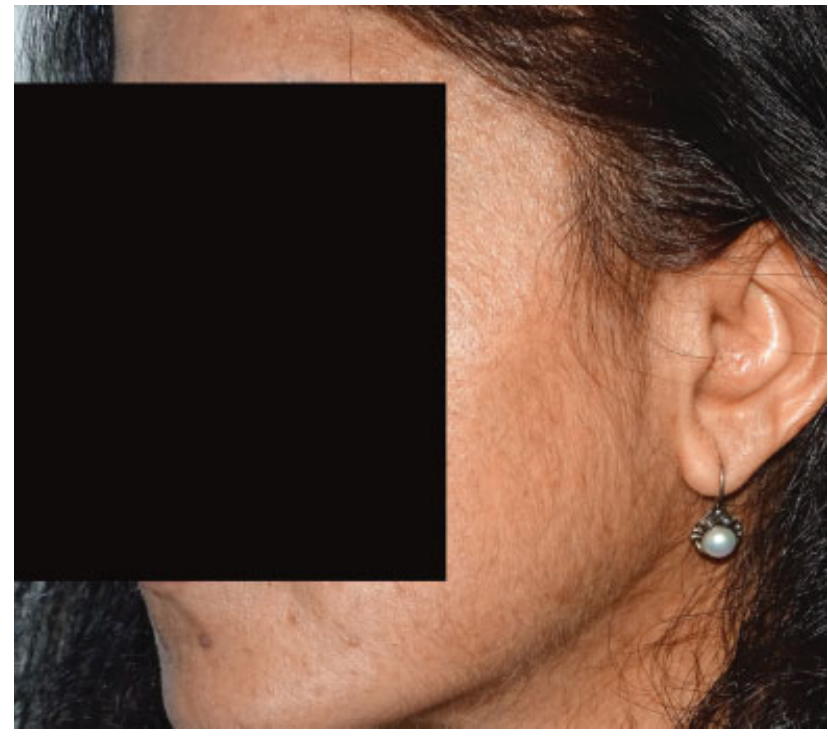

Fig. 6 Increase in facial hair after minoxidil application.

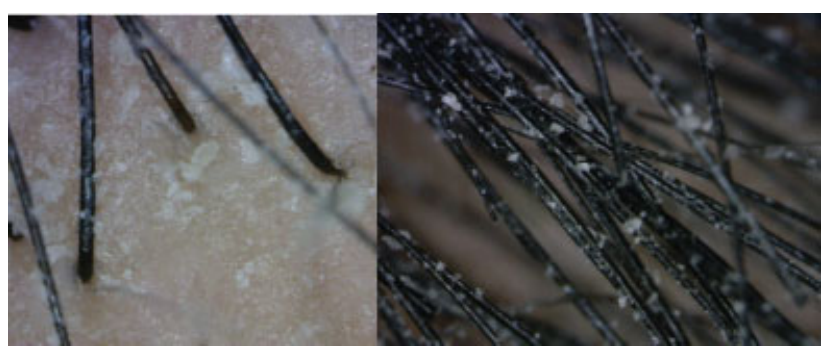

Fig. 7 Minoxidil crystal precipitation on hair

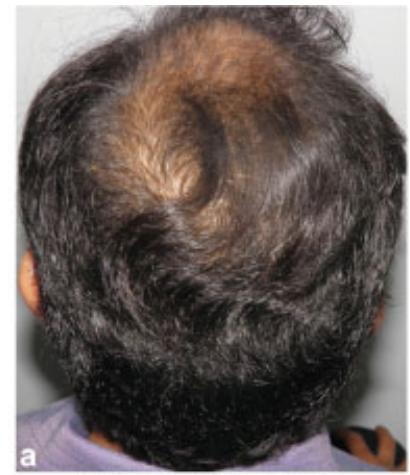

Before Minoxidil Application

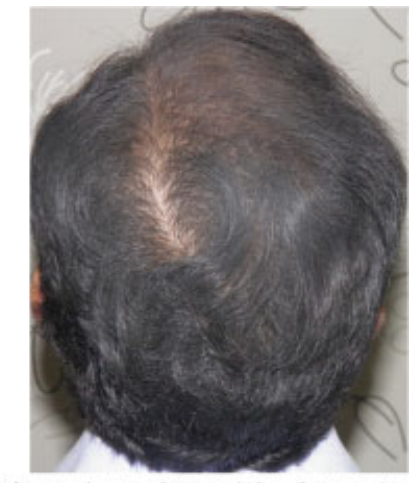

After regular use of Minoxidil for a few month Dull looking hair with loss of shine

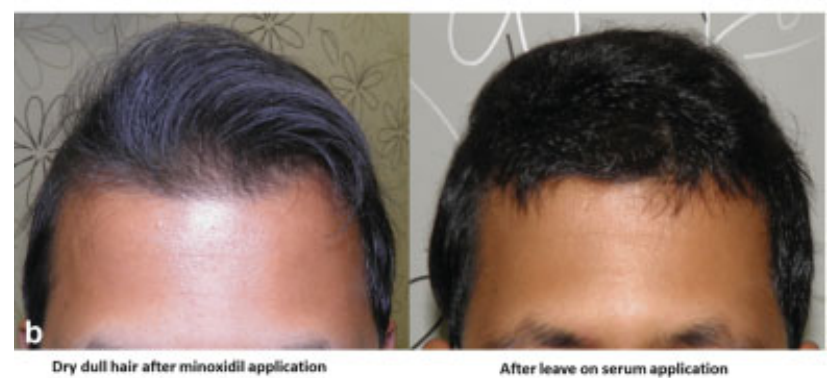

Fig. 8 (a) Dull look after minoxidil application. (b) Addressing dull look after minoxidil application with leave on serum. 
mon, some patients do complain of headaches. ${ }^{26}$ Lowering the dose helps in these cases.

\section{How to Use Topical Minoxidil}

For patients to reap benefits out of the use of topical minoxidil, they need to ensure correct application of minoxidil. It is helpful to demonstrate to the patient the correct way to apply minoxidil or direct them to videos which demonstrate the same. Minoxidil is for application to scalp, and for this, the scalp has to be dry.

Minoxidil is applied to the scalp as a liquid or foam, ideally right after bathing, so it is not washed away from towel-dried scalp (one need not blow dry it, it is okay if it is slightly damp). Maximum efficacy requires application two times daily to the affected area for men; one time daily for women (if using 5\%). No other application should be made on the scalp for at least 4 hours after minoxidil application. ${ }^{36}$ There is no need to massage it onto the scalp. It is important to avoid the eyes and make sure to wash hands after application.

Its off-label uses are found in the following area: alopecia areata, beard augmentation, eyebrow augmentation, postchemotherapy hair fall and telogen effluvium, ${ }^{26}$ and some have recommended for preoperative use on chest to get more follicles in anagen phase before harvesting them with follicular unit extraction (FUE). ${ }^{37}$

\section{Minoxidil Combinations and Formulations}

Significant irritation caused by PG have prompted various attempts to overcome these issues by replacing PG and alcohol with alternative solvents. PG-free preparations were designed (PG-free foam) in a bid to reduce the irritation and ensure patient compliance to the treatment. ${ }^{26,38}$ As a personal experience, the authors have observed that the stability of locally available minoxidil foam preparations in tropical climate is not optimal and the minoxidil containers need to be refrigerated before use.

Minoxidil absorption is known to increase with the concentration of minoxidil, but studies comparing $5 \%$ minoxidil with $10 \%$ found that the former was superior to the latter in hair regrowth and caused less irritation at the same time. ${ }^{39}$ The increased irritation does not justify usage of higher concentration, and the authors do not recommend usage of strengths higher than $5 \%$.

Attempts have been made to add minoxidil with other pharmacological actives and not always successfully. ${ }^{40}$ Tretinoin is thought to increase the absorption of minoxidil and also upregulate the expression of sulfotransferase, but studies have revealed that using a combination of minoxidil $5 \%+$ tretinoin $0.01 \%$ once a day was not superior to minoxidil $5 \%$ twice a day. This combination can cause significant irritation and may also interfere with minoxidil absorption. There is a possibility that if retinoic acid is applied a couple of hours before minoxidil application, then it may prove beneficial, but more studies are needed on the same. ${ }^{34}$

Topical combination of minoxidil with finasteride is discussed in the following section.

\section{Oral Minoxidil}

The minoxidil story seems to be coming to a full circle now with studies exploring the potential use of oral minoxidil $(\mathrm{OM})$ in treating hair loss. The proponents of OM have rationalized its use in this form to overcome the disadvantages of topical applications, namely, scalp irritation, daily twice application, unpredictable outcomes, etc. ${ }^{41,42}$ Different dosing regimens have been utilized in scarring and nonscarring alopecia, varying from 0.25 to $5 \mathrm{mg}$ daily with women requiring lower doses than men. ${ }^{42}$ However, the studies have shown that efficacy of $1 \mathrm{mg} \mathrm{OM}$ is similar to $5 \%$ topical minoxidil. The adverse effects are seen in fair number of patients (20\%) using OM, of which hypertrichosis is the most frequent (significant enough to mandate laser hair reduction). ${ }^{43}$ In the authors' opinion, the risk benefit ratio of OM as compared with topical minoxidil and the fact that the available evidence for OM is not of very high quality and does not justify use of OM routinely in the management of PHL and that topical minoxidil is still the first-line therapy. ${ }^{26,43,44}$ More studies would be needed to elucidate safety and efficacy of OM and justify its use as the first-line therapy in PHL. As a strict off-label indication with detailed informed consent, and after proper cardiac evaluation, it could be considered in motivated young healthy adults with no comorbidities who do not tolerate topical minoxidil or see benefits in spite of proper and diligent usage. ${ }^{45,46}$

\section{Finasteride}

As with minoxidil, serendipity once again played a role in identifying finasteride as a treatment for MPHL, when it was observed to promote hair growth in bald men who were taking it for benign prostate hyperplasia (BPH), leading to its approval by US Food and Drugs Administration (FDA) in 1997 for the treatment of MPHL (androgenic alopecia [AGA]). ${ }^{8}$

\section{How does Finasteride Work in MPHL}

What we know about androgens and MPHL

- There is increased expression of ARs, $5 \alpha$ reductase type 2 enzyme (SRD5A2) and dihydrotestosterone (DHT) in the follicles of the balding regions.

- There is decrease aromatase enzyme expression in the follicles of balding scalp.

- Blocking conversion of testosterone (T) to DHT delays or prevents progress of hair loss in MPHL. ${ }^{47-52}$

The contributory role of androgens in FPHL is still not clear. ${ }^{52,53}$ The role of androgens in FPHL becomes significant if there is presence of clinical hyperandrogenism (hirsutism, weight gain, irregular menses, difficulty in conceiving) or evidence of biochemical excess of androgens. Detailed assessment to rule out hyperandrogenism is a must in patients with FPHL, which includes testosterone, free and total, DHT, dehydroepiandrosterone sulfate (DHEAS), prolactin and sex hormone-binding globulin. ${ }^{51}$

Steroid type II $5 \alpha$-reductase (SRD5A2), an intracellular enzyme present in the outer root sheath in the DP of balding follicles, converts T into DHT, which is the preferred ligand for $A R .{ }^{54} \mathrm{DHT}$ binding to the AR puts into motion a 
cascade of events, which ultimately leads to hair follicle miniaturization.

Finasteride, a synthetic 4-azasteroid compound, is a specific and competitive inhibitor of SRD5A2, which converts T into $5 \alpha$ DHT. $^{55,56}$ Finasteride is known to reduce the scalp and serum DHT levels by 60 to $70 \%{ }^{51,54}$ Various studies of finasteride $1 \mathrm{mg}$ orally taken daily have shown cessation of hair loss progression and improved hair growth in a significant number of patients. Finasteride usage in MPHL has shown to increase the duration of anagen with increase in hair count, improved scalp coverage, and increased hair mass. ${ }^{1,56}$ Finasteride does not affect $\mathrm{T}$ levels or binding of DHT to AR and therefore does not possess androgenic, antiandrogenic, estrogenic or antiestrogenic, or progestational or steroid hormone-related properties. ${ }^{56,57}$

\section{Pharmacokinetics}

Finasteride when taken orally has a mean half-life of approximately 6 hours, mandating a daily dosage to maintain uniform blood levels. Extensive hepatic metabolism is by the cytochrome P450 enzyme system, specifically CYP3A4 enzyme subfamily. Finasteride does not have any major drug interactions ${ }^{56,58}$ but drugs like erythromycin or rifampicin, which impact CYP3A4 enzyme, should be used carefully with finasteride, and the authors recommend evaluation of liver function before starting treatment. ${ }^{57}$ Finasteride is recommended in the dosage of $1 \mathrm{mg}$ daily for treatment of MPHL. Although various dosages ranging from $0.01 \mathrm{mg}$ to $5 \mathrm{mg}$ /day have been tried, $1 \mathrm{mg}$ dosage has been found to be optimal. ${ }^{57}$

Finasteride has level 1 evidence for efficacy to prevent progression and improve hair loss in MPHL. 1,4,19,20,32,54,56-59 Studies have revealed that the hair count and weight start show increase by approximately 6 months and the improvement continues at least for up to 5 years. ${ }^{20,57}$ Treatment started earlier in the course of hair loss always responds better (-Figs. 9, 10, 11).

The authors have routinely used a combination of topical minoxidil and oral finasteride as an adjunct to hair transplant surgery to tackle the dynamic and static aspects of MPHL. When used preoperatively (at least 12-16 weeks), it helps to improve yield by prolonging anagen and also helps to reduce postoperative telogen effluvium. When used postoperatively, medications help to improve the vellus hair present

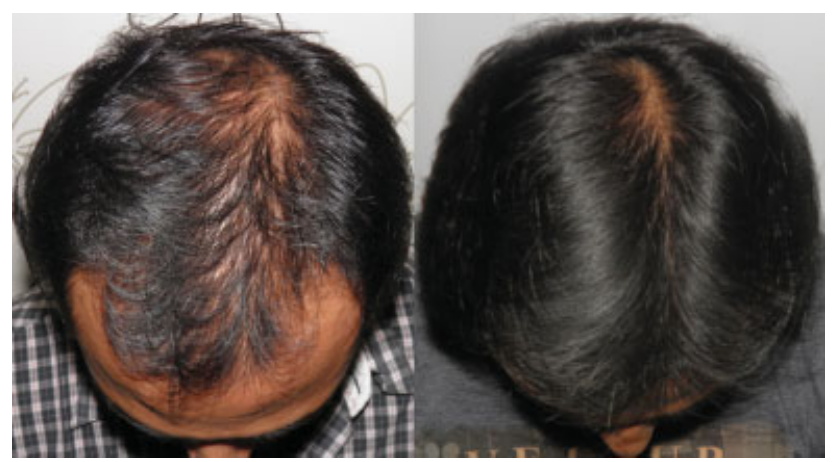

Fig. 9 Results of using topical minoxidil and oral finasteride.

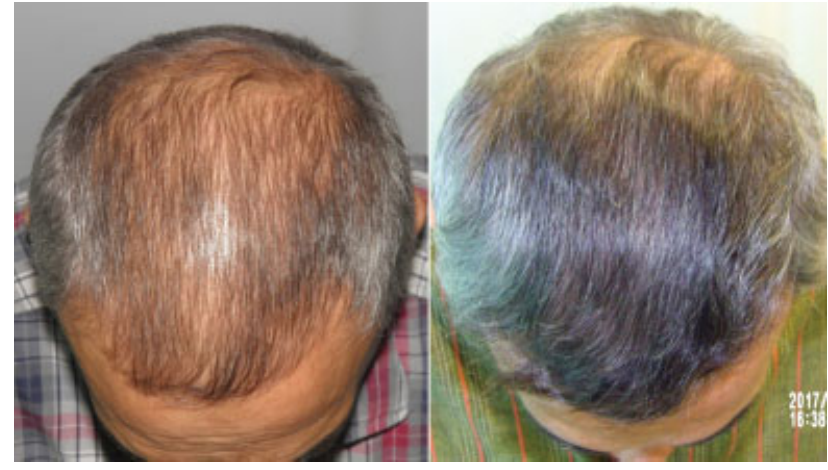

Fig. 10 Results of using topical minoxidil and oral finasteride.

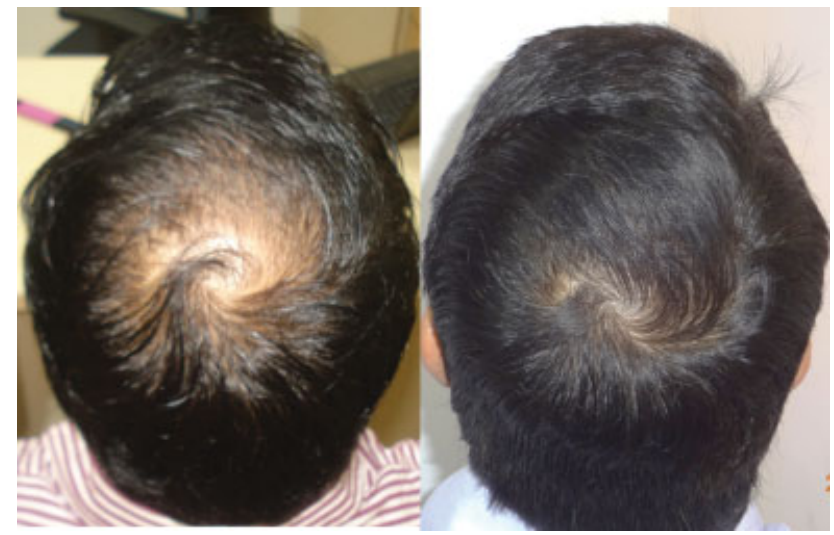

Fig. 11 Results of using topical minoxidil and oral finasteride.

in the transplanted and surrounding areas. Improvement of these hairs not only stabilizes hair loss progression but also augments hair transplant outcomes. This approach goes a long way in optimizing donor supply over patient's lifetime and increasing the interval between two transplants. ${ }^{9,10,60,61}$ When planning a transplant, it is important to put these considerations across to the patient and emphasize the benefits of a holistic approach (-Fig. 12).

\section{Adverse Effects of Oral Finasteride}

Finasteride has been widely used since 1992 for BPH and 1997 for MPHL, and millions of patients having used it and benefitted from it since then. This is a reflection of its safety and efficacy. However, the controversy surrounding the adverse effects continues, confounding the doctors as well as patients about its role in therapy.

The adverse effects related to finasteride usage can be divided into sexual (reduced libido, erectile dysfunction [ED], ejaculatory problems) and nonsexual (breast tenderness and enlargement; depression; hypersensitivity reactions including rash, pruritus, urticaria, and swelling of the lips and face; and testicular pain).

The widespread information or misinformation on the Internet regarding the potential adverse effects of finasteride, along with the fact that United States National Institutes of Health added a link for postfinasteride syndrome to its Genetic and Rare Disease Information Centre, ${ }^{62}$ has created a 

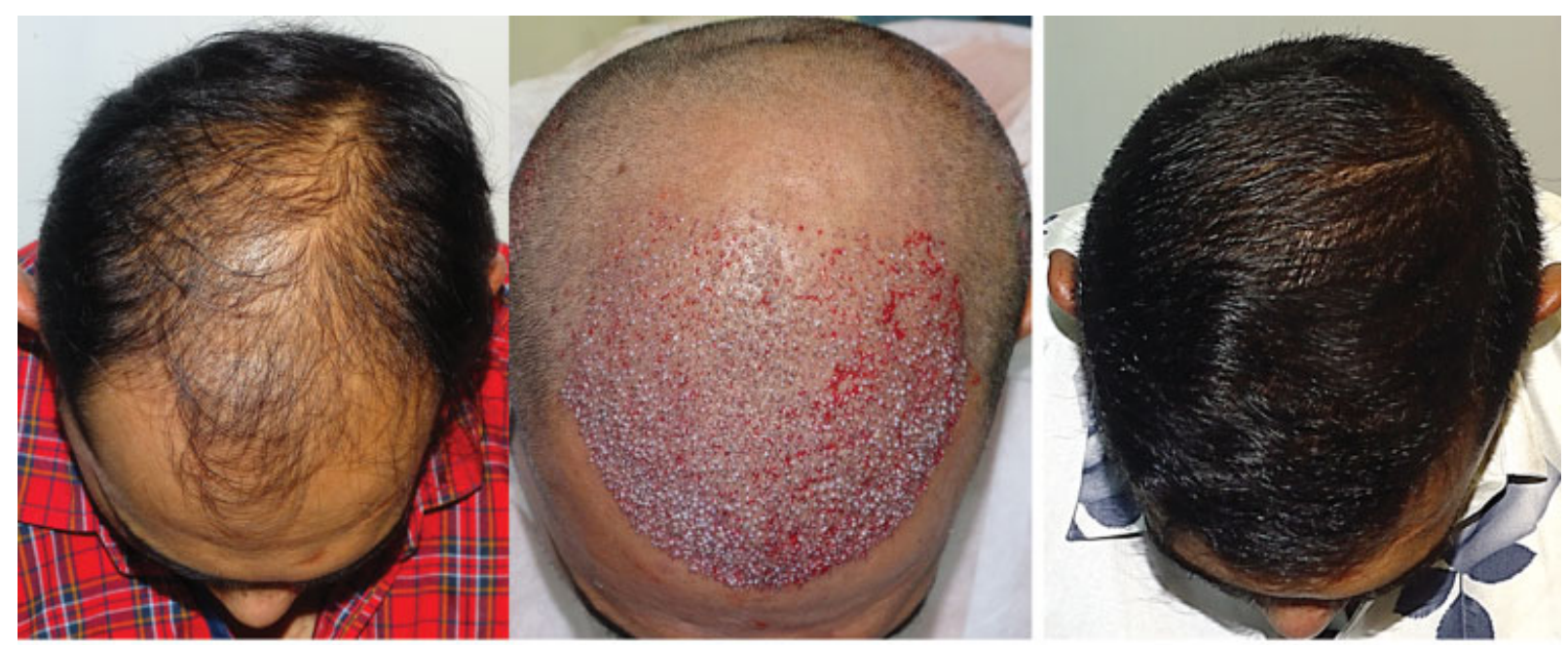

Results showing improved scalp coverage after combining hair transplantation for frontal and mid-scalp with medications addressing the thinning in the vertex region

Fig. 12 Showing beneficial effects of oral finasteride and topical minoxidil in hair transplant outcomes.

bias in the minds of patients as well as doctors who are very reluctant to use this medication.

The incidence of sexual adverse effects with SRD5A inhibitors quoted in literature range from 0.9 to $38 \%{ }^{62}$ of the sexual adverse effects, ED is more common than ejaculatory dysfunction and loss of libido. ${ }^{1,62}$ Literature on adverse effects of finasteride includes different doses, different drugs (finasteride and dutasteride) with different indications (BPH and MPHL), and different age groups. There are some publications which link finasteride usage with sexual adverse effects $^{62-67}$ and others which state that the association is not significant or does not exist. ${ }^{68-73}$ More importantly, metaanalysis of data regarding finasteride adverse effects in MPHL is not associated with statistically significant incidence. ${ }^{68-70}$ This could be probably due to the lower dosage used and the younger study population.

ED has two components-organic (levels of androgens and normal anatomy of genitals) and psychogenic, and both of these components may have an intricate relationship. ${ }^{70}$ Finasteride lowers serum DHT without altering T levels which, due to its humoral endocrine and local paracrine effects, is more relevant or critical to maintaining erectile function than the locally acting DHT. ${ }^{68,74}$ Most studies emphasize the importance of $\mathrm{T}$ in erectile physiology, which is highlighted by the fact that patients with congenital deficiency of SRD5A have normal erectile physiology. ${ }^{68,74,75}$ On a subjective level, patients answering the International Index of Erectile Function questionnaire showed no difference in scores before and 6 months after starting finasteride $1 \mathrm{mg}{ }^{76,77}$ Another factor is that hair loss may itself cause depression, lead to sexual problems, and confound the occurrence of ED.

From a psychogenic perspective, one possible explanation for the sexual adverse effects could be the "nocebo effect," which is not directly related to the specific pharmacological effect of the drug, but due the information about the drug being given during counselling (especially sexual side effects with finasteride). ${ }^{62,73}$ The art of counselling is to develop innovative ways to reduce nocebo effects without withholding information or scaring the patient. ${ }^{78}$ In practice, the authors navigate this difficult issue by sharing patient experiences and emphasizing the positive outcomes of finasteride with before and after pictures, downplaying the expectations of adverse effects (without distorting facts), being empathetic to the patient's situation and, most importantly, answering all their queries patiently. An effort is made to get the history regarding any existing sexual or psychiatric problems faced by them. If the patient has history of any sexual problems or psychological issues, starting finasteride is avoided or deferred.

Another adverse issue linked to finasteride is depression. Over the years, increased usage and subsequent postmarketing feedback have been responsible for adding depression to the list of adverse symptoms in the package insert of finasteride. ${ }^{62}$ SRD5A enzyme is not only responsible for reduction of T to DHT but also progesterone and deoxycorticosterone to dihydroprogesterone (DHP) and dihydrodeoxycorticosterone (DHDOC), respectively. $3 \alpha$ reduction converts DHT, DHP and DHDOC to steroid metabolites, which act on the gamma-aminobutyric acid (GABA) A receptors. The hypothesis for the cause of depressive symptoms occurring with finasteride therapy is linked to reduced levels neuroactive steroids, caused by SRD5A inhibition. Levels of neuroactive steroids have been found to be inversely proportional to various psychiatric conditions including depression. ${ }^{79}$ Animal studies have yielded evidence which supports the fact that finasteride is a depressogenic drug, but human studies have not corroborated the same. This could be due to species difference in finasteride activity or the accuracy of the modalities used to assess depression-like symptoms in animals. ${ }^{79}$ Recent publications however have found the risk of developing depression after finasteride therapy to be low and not statistically significant. ${ }^{80,81}$ However, more studies would be needed to validate these findings and till then it 
would be a good practice to rule out any preexisting psychiatric issues in the patient and also consider and discuss the possible sexual side effects and risk of depression with patients prior to starting finasteride therapy. ${ }^{65}$

\section{Finasteride and Prostate Cancer}

The Prostate Cancer Prevention Trial (PCPT) results showed that finasteride users were $25 \%$ less likely to develop cancer of the prostate. But the controversial finding was that the incidence of high-grade cancers was $0.7 \%$ in finasteride users. ${ }^{82,83}$ In view of these findings, the FDA subsequently in 2011 issued a safety warning of developing high-grade cancer as an adverse effect of finasteride. ${ }^{83}$ Various studies conducted later as well as further analysis of the PCPT outcomes by factoring in the biases and accounting for PSA (increased sensitivity), showed that finasteride usage is not linked to increased incidence of high-grade prostate cancers. ${ }^{83-86}$

Prostate-specific antigen (PSA) is an important tumor marker for prostate cancer, and finasteride is known to decrease the levels. There have been various recommendations about interpreting PSA levels in patients taking finasteride. As the levels are known to decrease, a compensatory adjustment of the PSA concentration (to multiply the value by two) is recommended to get the corrected value. ${ }^{87,88}$ However, this correction is not valid for long-term management, and any increase above the baseline is to be viewed with suspicion even if it is in normal range. ${ }^{89}$ The authors routinely advise PSA estimation in all male patients above the age of 45 years who are agreeable to start with finasteride. The test is then repeated after 6 months of therapy and then annually. The 6-month value is taken as the new baseline and any change in that value, even if it is in the normal range, is taken seriously and further investigations advised.

\section{Finasteride and Male Breast Cancer}

A report published by Medicines and Health care products Regulatory Agency (MHRA), United Kingdom, in 2009 mentioned the occurrence of male breast cancer (MBC) in patients who received finasteride. ${ }^{90}$ Another study in 2018 also suggested possible association between finasteride use and MBC but added that this could also be attributed to the increased surveillance and the way data was collected (ascertainment bias), which could confuse the inference. ${ }^{91}$ In 2019, a large case-control study using individual-level registry data from Denmark, Finland, and Sweden, which adjusted for confounding factors, showed that there is no association between finasteride usage and $\mathrm{MBC} .^{92}$

\section{Finasteride and Fertility}

A randomized, double-blind placebo-controlled study, which reviewed the effect of $1 \mathrm{mg}$ finasteride on fertility in young men, showed no significant effects on sperm concentration, total sperm per ejaculate, sperm motility, or morphology. $58,93,94$

However, a similar study using $5 \mathrm{mg}$ dose did show a statistically significant reduction in sperm count, sperm motility, semen volume, and sperm concentration from baseline but no change in sperm morphology. These changes however were not statistically significant at 52 weeks. Moreover, these changes reverted to baseline after discontinuing treatment. ${ }^{74}$ Another study conducted in the male infertility population showed improved semen parameters, including increased sperm count after discontinuation of finasteride. ${ }^{95}$ This probably indicates that finasteride may not significantly impact sperm parameters, but in patients with conditions related to infertility, an amplification of the negative influence of finasteride could occur. ${ }^{96}$ Keeping this in mind, the authors defer finasteride therapy in young men who are planning to start their family, especially if the couple is having difficulty in conceiving.

\section{Postfinasteride Syndrome}

Postfinasteride syndrome (PFS) is a collection of adverse effects causing sexual, neuropsychiatric, and physical symptoms which develop with use of finasteride for MPHL or BPH and which persist after discontinuation of treatment. ${ }^{97-99}$ Majority of the studies which reported on PFS were found to be of low quality, having come from doctors who are not treating hair loss, and most of them suffered from a strong selection bias and nocebo effect. ${ }^{100}$ Although most individuals who suffer from PFS have homogenous symptoms, the medical community is still not recognizing this as a medical condition, as most reports cannot deny nor confirm its existence as a nosological entity. ${ }^{98,101}$ Nevertheless, it has been added to the Genetic and Rare Disease Information Centre by the United States National Institutes of Health. ${ }^{62}$ One of the studies has labeled PFS as a delusional disorder of the somatic type, in a person with probably a personality disorder, with the potential of a mass psychogenic illness due to its media coverage. ${ }^{101}$ Most observations pertaining to PFS are mainly based on self-reporting of symptoms by the patients, and limited medical studies have not been able to identify molecular mechanisms and/or genetic determinants underlying these adverse effects. ${ }^{99}$ The authors have not seen a single case of persistent adverse effects with finasteride therapy in a practice over two decades, but they make it a point to watch out for the red flags like a history of preexisting psychological problems such as depression or personality disorders before choosing to start with finasteride therapy.

\section{Topical Finasteride}

Although oral finasteride is approved for treatment of MPHL due to the incidence of small but significant number of adverse effects and reluctance of patients to use oral finasteride; topical finasteride was explored as an alternative. Topical finasteride is an off-label use of finasteride for MPHL. In India, it is available as a combination $0.1 \%$ finasteride with minoxidil 5\%. ${ }^{102,103}$ The study by Casserini et al compared pharmacokinetics of topically applied finasteride with the standard oral finasteride $1 \mathrm{mg}$. The plasma DHT suppression was almost the same with topical and oral forms of finasteride, but at the same time, the maximum plasma concentration (Cmax) of finasteride was much 


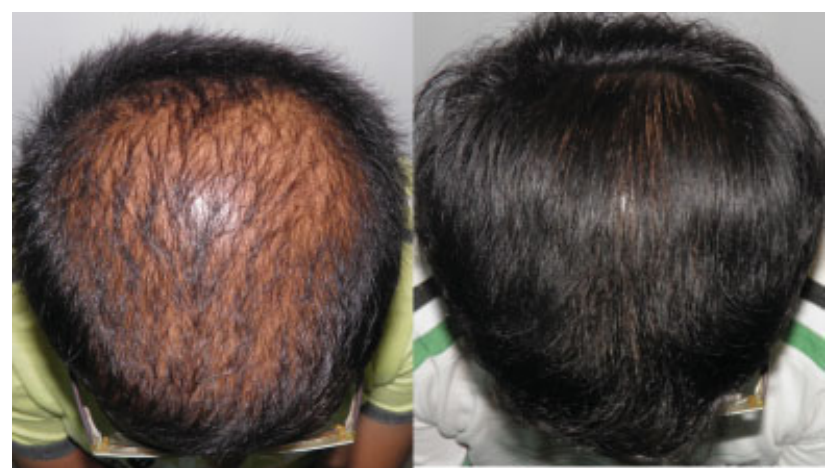

Fig. 13 Improved scalp coverage with topical finasteride and minoxidil.

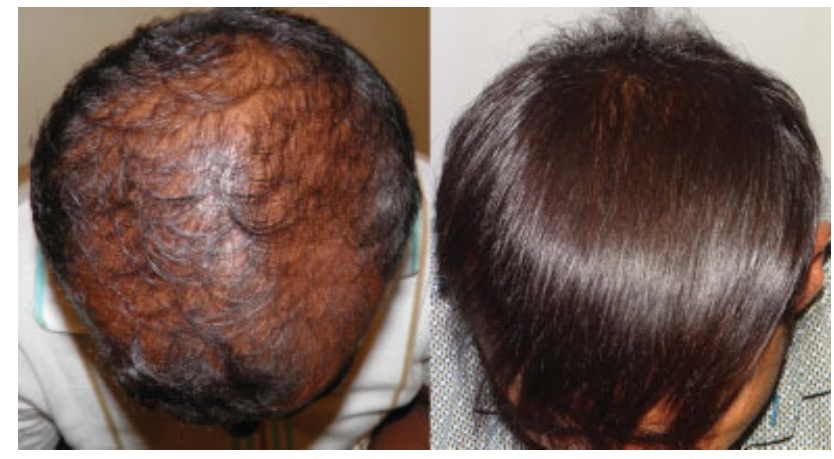

Fig. 14 Improved scalp coverage with topical finasteride and minoxidil.

lower with topical finasteride. ${ }^{104}$ So, in effect an equal therapeutic benefit was achieved without increasing the systemic exposure to finasteride; therefore, potentially minimizing the incidence of adverse effects. The benefits in terms of hair growth are comparable to oral finasteride (-Figs. 13, 14, 15). ${ }^{102,103,105}$

\section{Dutasteride}

Dutasteride, a potent type I and type II $5-\alpha$ reductase inhibitor, and the logic of using this is that dual $5 \alpha$-reductase inhibition could result in greater efficacy than is observed in selective type 2 inhibition, and this dual inhibition could prevent type 1-mediated synthesis of $\mathrm{DHT}^{106}$ It is approved for BPH but is also prescribed as an off-label treatment for pattern hair loss. ${ }^{1,107}$ It has a high level of evidence for safety and efficacy, as per the Japanese guidelines. ${ }^{20}$ It is thought to be more potent than finasteride, as it can decrease serum DHT by more than $90 \%$, while finasteride decreases serum DHT by $70 \%{ }^{54}$ Different studies compared dutasteride versus finasteride to assess its efficacy and safety. It has been found that dutasteride is slightly more efficacious than finasteride and the adverse effect profile is better than finasteride. ${ }^{107,108}$ The dosage used is $0.5 \mathrm{mg}$ daily. As dutasteride has a much longer half-life and there is limited data of long-term safety, the authors do not use it as a first-line treatment in MPHL. The authors offer it as an off-label drug to patients who have plateaued in their response to finasteride or have not responded to finasteride only after proper counselling.

\section{Antiandrogens}

FPHL with evidence of clinical or biochemical androgen excess would be suitable for antiandrogen therapy as compared with or without any evidence of hyperandrogenism. ${ }^{19}$ Hormonal treatment for FPHL can be divided into two broad groups, antiandrogens and estrogenic (or antiestrogenic) drugs. ${ }^{4}$ Two commonly used drugs are cyproterone acetate and spironolactone, although evidence of efficacy for any of these treatments is limited. ${ }^{54}$

\section{Spironolactone}

It is by far the most common antiandrogen used off-label in FPHL. $^{1,4}$ Spironolactone is a potassium-sparing diuretic, structurally related to aldosterone (antagonist), which acts as an antiandrogenic by competitively inhibiting the androgen receptor in target tissues and also inhibits ovarian production of androgens, reducing the levels of total $\mathrm{T}$ and competitively blocking the AR..$^{1,4,54}$ The dosage ranges from $50 \mathrm{mg} /$ day to $200 \mathrm{mg} /$ day for at least 6 months. Some patients may face adverse effects like postural hypotension and electrolyte imbalance. ${ }^{4}$ It is contraindicated in MPHL.

\section{Cyproterone Acetate}

Cyproterone acetate (CA) is an AR blocker and potent progesterone derivative that prevents dihydroxytestosterone from binding to the AR and inhibits release of folliclestimulating hormone (FSH) and luteinizing hormone (LH), thereby reducing testosterone levels. ${ }^{1,4,54} \mathrm{CA}$ is not approved in the United States. It is available combined with ethynyl estradiol as an oral contraceptive in many countries ( $2 \mathrm{mg}$ CA with ethinyl estradiol $0.035 \mathrm{mg}$ ) and started on day 1 of the menses for 21 days, followed by a break of 7 days. Studies have shown statistically significant improvement in hair

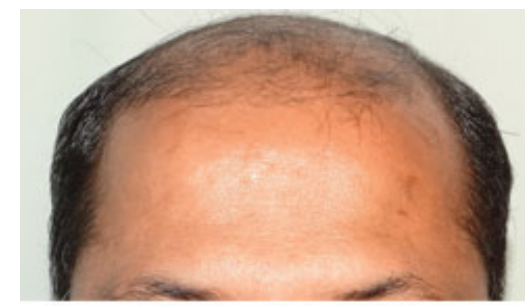

PRE TREATMENT

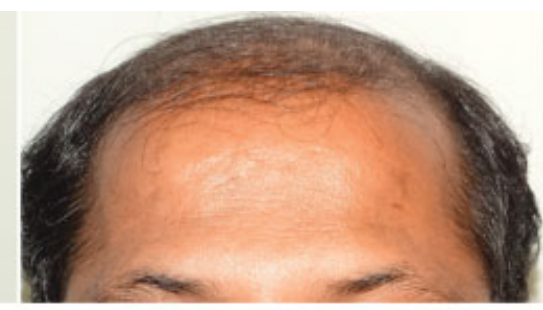

6 months after TOPICAL MINOXIDIL 5\%

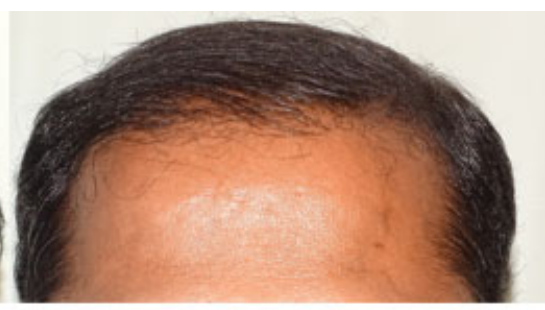

6 months after changing to TOPICAL FINASTERIDE + MINOXIDIL

Fig. 15 Improvement with minoxidil and later with minoxidil and finasteride combination 
growth as compared with placebo. ${ }^{4}$ It is important to keep a track of liver function, as it can cause liver toxicity. The authors have used it in patients of FPHL who also have polycystic ovarian disease (PCOD).

\section{Prostaglandin Analogues}

The beneficial effects of prostaglandin analogue on hair growth were noted when eyelashes improved with topical use for treatment of glaucoma. PGD2 levels are associated with miniaturization in balding scalp while levels of PGE2 and PGF2 are reduced. ${ }^{59}$ PGF2 analogues bimatoprost and latanoprost are known to promote hair growth. But currently there is insufficient evidence to use them in PHL. ${ }^{4,20}$

\section{Ketoconazole}

Ketoconazole is an antifungal agent available as a shampoo in combination with zinc pyrithione. It is thought to have local DHT-blocking effects as well as an anti-inflammatory effect. ${ }^{1,4,20}$ Furthermore, change in the microbiome of the hair follicle, causing microinflammation in the perifollicular region, is thought to be one of the factors implicated in PHL. ${ }^{1,59}$ Use of ketoconazole with its antifungal properties and antiinflammatory properties of zinc pyrithione has worked well in conjunction with topical minoxidil. ${ }^{59} \mathrm{~A}$ few noncontrolled studies have shown benefits in MPHL as well as in FPHL with androgen excess. ${ }^{1,4,20,59}$

\section{Saw Palmetto (Serenoa repens)}

Saw palmetto (SP), a botanical extract from the berries of Serenoa repens, is one among the many naturally occurring SRD5A inhibitors which has gained popularity as a magical remedy for MPHL. ${ }^{59,109,110}$ Very few studies of efficacy of SP in PHL exist and most of the data on SP is from studies of its use in $\mathrm{BPH} .{ }^{110}$ It is listed in US Pharmacopeia under food supplements and available as dried berries or tablets of the extract. The dosage is $160 \mathrm{mg}$ twice a day. ${ }^{109}$ In absence of strong evidence of efficacy, it cannot be recommended.

Other botanicals which have been proposed to be used in PHL are green tea ( $C$ sinensis), pumpkin seed ( $C$ pepo), rosemary (Rosmarinus officinalis), grape seed (Vitis vinifera), and licorice (G glabra). These do not have supporting data to recommend their use in PHL.

\section{Peptides}

Botanical extracts have been used to treat PHL, especially biomimetic peptides such as acetyltetrapeptide-1 and 3, which are combined with an isoflavone derived from red clover (biochanin-A). There is very little literature support, and their role in management of PHL is not significant. Probably, they may play a role of placebo.

\section{Nutritional Supplements}

The hair follicle is a miniature organ with a high cellular turnover and a high metabolic rate, which mandates a constant supply of energy and nutrients. So, obviously nutrient deficiencies will impact structure and growth of hair. ${ }^{111,112}$ The kind of hair loss seen with nutrient deficiencies is generally of a diffuse variety and does not have the typical characteristics of pattern hair loss. Nutrient supplementation has been used empirically to address hair loss concerns, with many patients using OTC products to manage hair loss. ${ }^{111-113}$ However, the role of nutrient deficiencies in causation of pattern hair loss has never been really verified. ${ }^{112}$ However, in patients of pattern hair loss who have coexisting deficiencies of certain nutrients like vitamin D or iron, supplementing these can improve the outcomes in pattern hair loss. ${ }^{113}$ Data on the role of nutrient supplementation in the absence of deficiency is extremely limited. $^{112}$

\section{Summary}

The goal of the pharmacological management of PHL is to prevent further progression of hair loss and promote hair regrowth and improve scalp coverage. The key to successfully management of PHL using medications is proper diagnosis, adequate patient counselling, regular periodic follow-up, watching for adverse issues, and constant reassurance to the patient. The success of the treatment is to a large extent controlled by patient compliance and ensuring this is important. Emphasizing the following points is critical for its success:

1. That PHL is a condition that is going to progress with time and medical management would be necessary either alone or in conjunction with hair transplantation.

2. It is critical to give the patient all options of dealing with his or her hair loss, explaining the pros and cons of each approach. With all the options advertised on the Internet and social media, patients tend to be overwhelmed and confused. Patience and empathy along with clear recommendations are helpful to the patient, especially young men who are in denial and do not accept medical therapy and would rather proceed with hair transplantation.

3. It is important to mention the benefits and the adverse effects without highlighting them; periodic photographic documentation of the progress is crucial and always helps the patient to stay on track with the treatment. Important to point out that majority of the patients do not encounter adverse effects.

4. Medical therapy is best started early, used daily, and continued for life.

5. Currently, there are only two approved medications to achieve this along with off-label uses of other medications, but in terms of efficacy and safety, only topical minoxidil and oral finasteride in MPHL and topical minoxidil for FPHL have the evidence to support their use.

6. However, it is important to wait for at least 6 months before expecting and visible change. It is ideal to combine minoxidil and finasteride in MPHL as they work synergistically.

7. Pharmacotherapy plays an important role in the hair transplant setting. Use of medications perioperatively helps to optimize the amount of donor harvest and at the same time augments the results of the transplant, thereby conserving the donor area for future needs. 
8. Initial hair fall occurring with treatment should not be a cause of worry and usually settles in 8 to 10 weeks. It is always helpful to educate the patient on the proper technique of minoxidil application.

9. Cosmetic management for dealing with dry and dull hair after minoxidil application must be shown to the patient.

10. Sexual side effects after oral finasteride therapy, if they do occur, are usually seen after at least 6 months of therapy. It is important to reassure the patient. If they are not severe, it is okay to continue with the finasteride after counselling or may be discontinued with the understanding that the hair loss will return to pretreatment stage. There is also the option of using topical finasteride with minoxidil in apprehensive patients.

Conflict of Interest

None declared.

\section{References}

1 Tamashunas NL, Bergfeld WF. Male and female pattern hair loss: treatable and worth treating. Cleve Clin J Med 2021;88(03): 173-182

2 Cotsarelis G, Millar SE. Towards a molecular understanding of hair loss and its treatment. Trends Mol Med 2001;7(07):293-301

3 Higgins CA, Christiano AM. Regenerative medicine and hair loss: how hair follicle culture has advanced our understanding of treatment options for androgenetic alopecia. Regen Med 2014;9(01):101-111

4 Varothai S, Bergfeld WF. Androgenetic alopecia: an evidence-based treatment update. Am J Clin Dermatol 2014;15(03):217-230

5 Campo D, D'Acunzo V. Doctors and baldness: a five thousand year old challenge. G Ital Dermatol Venereol 2016;151(01):93-101

6 Kandil H, El-Mohamdy M. Role of the hair in ancient Egypt. International Journal of Tourism and Hospitality Management 2018;1(01):77-95

7 Rongen GA, Marquet P, van Gerven JMA, et al. The scientific basis of rational prescribing. A guide to precision clinical pharmacology based on the WHO 6-step method. Eur J Clin Pharmacol 2020

8 Yesudian P. Serendipity in trichology. Int J Trichology 2011;3 (01):1-2

9 Avram MR, Cole JP, Gandelman M, et al; Roundtable Consensus Meeting of The 9th Annual Meeting of The International Society of Hair Restoration Surgery. The potential role of minoxidil in the hair transplantation setting. Dermatol Surg 2002;28(10):894 -900 , discussion 900

10 Leavitt M, Perez-Meza D, Rao NA, Barusco M, Kaufman KD, Ziering C. Effects of finasteride $(1 \mathrm{mg})$ on hair transplant. Dermatol Surg 2005;31(10):1268-1276, discussion 1276

11 Pantelireis N, Higgins CA. A bald statement - Current approaches to manipulate miniaturisation focus only on promoting hair growth. Exp Dermatol 2018;27(09):959-965

12 Trüeb RM, Henry JP, Davis MG, Schwartz JR. Scalp condition impacts hair growth and retention via oxidative stress. Int J Trichology 2018;10(06):262-270

13 Peyravian N, Deo S, Daunert S, Jimenez JJ. The inflammatory aspect of male and female pattern hair loss. J Inflamm Res 2020; 13:879-881

14 Blume-Peytavi U, Blumeyer A, Tosti A, et al; European Consensus Group. S1 guideline for diagnostic evaluation in androgenetic alopecia in men, women and adolescents. Br J Dermatol 2011; 164(01):5-15

15 Avital YS, Morvay M, Gaaland M, Kemény L. Study of the international epidemiology of androgenetic alopecia in young Caucasian men using photographs from the Internet. Indian J Dermatol 2015;60(04):419
16 Krupa Shankar D, Chakravarthi M, Shilpakar R. Male androgenetic alopecia: population-based study in 1,005 subjects. Int $\mathrm{J}$ Trichology 2009;1(02):131-133

17 Gupta AK, Ivanova IA. Analysis of YouTube hair loss treatment information: What makes for engaging content? Dermatol Ther (Heidelb) 2020;33(06):e14244

18 Gupta AK, Ivanova IA. Hair loss treatment information on Facebook: content analysis and comparison with other online sources. J Cosmet Dermatol 2021;20(02):577-583

19 Kanti V, Messenger A, Dobos G, et al. Evidence-based (S3) guideline for the treatment of androgenetic alopecia in women and in men - short version. J Eur Acad Dermatol Venereol 2018; 32(01):11-22

20 Manabe M, Tsuboi R, Itami S, et al; Drafting Committee for the Guidelines for the Diagnosis and Treatment of Male- and FemalePattern Hair Loss. Guidelines for the diagnosis and treatment of male-pattern and female-pattern hair loss, 2017 version. J Dermatol 2018;45(09):1031-1043

21 Stafford N, Kahn G. BMJ 2014;349:g7798

22 Rossi A, Cantisani C, Melis L, Iorio A, Scali E, Calvieri S. Minoxidil use in dermatology, side effects and recent patents. Recent Pat Inflamm Allergy Drug Discov 2012;6(02):130-136

23 Davies GC, Thornton MJ, Jenner TJ, et al. Novel and established potassium channel openers stimulate hair growth in vitro: implications for their modes of action in hair follicles. J Invest Dermatol 2005;124(04):686-694

24 National Center for Biotechnology Information. PubChem Compound Summary for CID 4201, Minoxidil. Accessed August 29, 2021 at: https://pubchem.ncbi.nlm.nih.gov/compound/Minoxidil

25 Gupta AK, Foley KA. 5\% Minoxidil: treatment for female pattern hair loss. Skin Therapy Lett 2014;19(06):5-7

26 Suchonwanit P, Thammarucha S, Leerunyakul K. Minoxidil and its use in hair disorders: a review. Drug Des Devel Ther 2019; 13:2777-2786

27 Choi N, Shin S, Song SU, Sung JH. minoxidil promotes hair growth through stimulation of growth factor release from adiposederived stem cells. Int J Mol Sci 2018;19(03):691

28 Trüeb RM. Pharmacologic interventions in aging hair. Clin Interv Aging 2006;1(02):121-129

29 Kwack MH, Kang BM, Kim MK, Kim JC, Sung YK. Minoxidil activates $\beta$-catenin pathway in human dermal papilla cells: a possible explanation for its anagen prolongation effect. J Dermatol Sci 2011;62(03):154-159

$30 \mathrm{Ng}$ KW. Penetration enhancement of topical formulations. Pharmaceutics 2018;10(02):51

31 Novak E, Franz TJ, Headington JT, Wester RC. Topically applied minoxidil in baldness. Int J Dermatol 1985;24(02):82-87

32 Chitalia J, Dhurat R, Goren A, et al. Characterization of follicular minoxidil sulfotransferase activity in a cohort of pattern hair loss patients from the Indian Subcontinent. Dermatol Ther (Heidelb) 2018;31(06):e12688

33 Sharma A, Goren A, Dhurat R, et al. Tretinoin enhances minoxidil response in androgenetic alopecia patients by upregulating follicular sulfotransferase enzymes. Dermatol Ther (Heidelb) 2019;32(03):e12915

34 Monselise A, Cohen DE, Wanser R, Shapiro J. What ages hair? Int J Womens Dermatol 2017;3(1, Suppl):S52-S57

35 Leenen FH, Smith DL, Unger WP. Topical minoxidil: cardiac effects in bald man. Br J Clin Pharmacol 1988;26(04):481-485

36 Ferry JJ, Shepard JH, Szpunar GJ. Relationship between contact time of applied dose and percutaneous absorption of minoxidil from a topical solution. J Pharm Sci 1990;79(06):483-486

37 Saxena K, Savant SS. Body to scalp: evolving trends in body hair transplantation. Indian Dermatol Online J 2017;8(03):167-175

38 Gupta AK, Charrette A. Topical minoxidil: systematic review and meta-analysis of its efficacy in androgenetic alopecia. Skinmed 2015;13(03):185-189 
39 Ghonemy S, Alarawi A, Bessar H. Efficacy and safety of a new $10 \%$ topical minoxidil versus $5 \%$ topical minoxidil and placebo in the treatment of male androgenetic alopecia: a trichoscopic evaluation. J Dermatolog Treat 2021;32(02):236-241

40 Santos AC, Pereira-Silva M, Guerra C, et al. Topical minoxidilloaded nanotechnology strategies for alopecia. Cosmetics 2020; 7(02):21

41 Randolph M, Tosti A. Oral minoxidil treatment for hair loss: a review of efficacy and safety. J Am Acad Dermatol 2021;84(03): 737-746

42 Villani A, Fabbrocini G, Ocampo-Candiani J, Ruggiero A, OcampoGarza SS. Review of oral minoxidil as treatment of hair disorders: in search of the perfect dose. J Eur Acad Dermatol Venereol 2021; 35(07):1485-1492

43 Ramos PM, Sinclair RD, Kasprzak M, Miot HA. Minoxidil 1 mg oral versus minoxidil 5\% topical solution for the treatment of femalepattern hair loss: a randomized clinical trial. J Am Acad Dermatol 2020;82(01):252-253

44 do Nascimento IJB, Harries M, Rocha VB, et al. Effect of oral minoxidil for alopecia: systematic review. Int J Trichology 2020; 12(04):147-155

45 Vañó-Galván S, Pirmez R, Hermosa-Gelbard A, et al. Safety of low-dose oral minoxidil for hair loss: A multicenter study of 1404 patients. J Am Acad Dermatol 2021;84(06): 1644-1651

46 Almohanna HM, Perper M, Tosti A. Safety concerns when using novel medications to treat alopecia. Expert Opin Drug Saf 2018; 17(11):1115-1128

47 Davey RA, Grossmann M. Androgen receptor structure, function and biology: from bench to bedside. Clin Biochem Rev 2016;37 (01):3-15

48 Hibberts NA, Howell AE, Randall VA. Balding hair follicle dermal papilla cells contain higher levels of androgen receptors than those from non-balding scalp. J Endocrinol 1998;156(01):59-65

49 Azzouni F, Godoy A, Li Y, Mohler J. The 5 alpha-reductase isozyme family: a review of basic biology and their role in human diseases. Adv Urol 2012;2012:530121

50 Kische H, Arnold A, Gross S, et al. Sex hormones and hair loss in men from the general population of northeastern Germany. JAMA Dermatol 2017;153(09):935-937

51 Sawaya ME, Price VH. Different levels of 5alpha-reductase type I and II, aromatase, and androgen receptor in hair follicles of women and men with androgenetic alopecia. J Invest Dermatol 1997;109(03):296-300

52 Ustuner ET. Cause of androgenic alopecia: crux of the matter. Plast Reconstr Surg Glob Open 2013;1(07):e64

53 Carmina E, Azziz R, Bergfeld W, et al. Female pattern hair loss and androgen excess: a report from the multidisciplinary androgen excess and PCOS Committee. J Clin Endocrinol Metab 2019;104 (07):2875-2891

54 Alves R. Androgenetic alopecia: a review and emerging treatments. Clin Res Dermatol Open Access 2017;4(04):1-13

55 McClellan KJ, Markham A. Finasteride: a review of its use in male pattern hair loss. Drugs 1999;57(01):111-126

56 Accessed August 29, 2021 at: https://www.accessdata.fda. gov/drugsatfda_docs/nda/2011/0207880rig1s017.pdf

57 Tumkur A, Ern C, Shuang C, et al. The role of finasteride in the management of androgenetic alopecia in male: a narrative review. Indian Journal of Pharmacy Practice. 2019;13:3-13. Doi: $10.5530 /$ ijopp.13.1.2

58 Zito PM, Bistas KG, Syed K. Finasteride. Treasure Island (FL): StatPearls Publishing; 2021

59 Sonthalia S. Hair restoration in androgenetic alopecia: looking beyond minoxidil, finasteride and hair transplantation. Journal of Cosmetology \& Trichology 2016;02(01):

60 Van Neste D, Fuh V, Sanchez-Pedreno P, et al. Finasteride increases anagen hair in men with androgenetic alopecia. $\mathrm{Br} \mathrm{J}$ Dermatol 2000;143(04):804-810
61 Kassimir JJ. Use of topical minoxidil as a possible adjunct to hair transplant surgery. A pilot study. J Am Acad Dermatol 1987;16(3 Pt 2):685-687

62 Hirshburg JM, Kelsey PA, Therrien CA, Gavino AC, Reichenberg JS. Adverse effects and safety of 5-alpha reductase inhibitors (finasteride, dutasteride): a systematic review. J Clin Aesthet Dermatol 2016;9(07):56-62

63 Lee S, Lee YB, Choe SJ, Lee WS. Adverse sexual effects of treatment with finasteride or dutasteride for male androgenetic alopecia: a systematic review and meta-analysis. Acta Derm Venereol 2019;99(01):12-17

64 Irwig MS. Persistent sexual and nonsexual adverse effects of finasteride in younger men. Sex Med Rev 2014;2(01):24-35

65 Fertig RM, Gamret AC, Darwin E, Gaudi S. Sexual side effects of 5- $\alpha-$ reductase inhibitors finasteride and dutasteride: A comprehensive review. Dermatol Online J 2017;23(11):13030/qt24k8q743

66 Traish AM, Hassani J, Guay AT, Zitzmann M, Hansen ML. Adverse side effects of $5 \alpha$-reductase inhibitors therapy: persistent diminished libido and erectile dysfunction and depression in a subset of patients. J Sex Med 2011;8(03):872-884

67 Arias-Santiago S, Camacho-Martínez FM. Adverse effects of 5alpha reductase inhibitor therapy in men with androgenetic alopecia: is there cause for concern? Actas Dermosifiliogr 2016; 107(09):709-711

68 Canguven O, Burnett AL. The effect of 5 alpha-reductase inhibitors on erectile function. J Androl 2008;29(05):514-523

69 Liu L, Zhao S, Li F, et al. Effect of $5 \alpha$-reductase inhibitors on sexual function: a meta-analysis and systematic review of randomized controlled trials. J Sex Med 2016;13(09):1297-1310

70 Shin YS, Karna KK, Choi BR, Park JK. Finasteride and erectile dysfunction in patients with benign prostatic hyperplasia or male androgenetic alopecia. World J Mens Health 2019;37(02):157-165

71 Gupta AK, Charrette A. The efficacy and safety of $5 \alpha$-reductase inhibitors in androgenetic alopecia: a network meta-analysis and benefit-risk assessment of finasteride and dutasteride. J Dermatolog Treat 2014;25(02):156-161

72 Haber RS, Gupta AK, Epstein E, Carviel JL, Foley KA. Finasteride for androgenetic alopecia is not associated with sexual dysfunction: a survey-based, single-centre, controlled study. J Eur Acad Dermatol Venereol 2019;33(07):1393-1397

73 Mondaini N, Gontero P, Giubilei G, et al. Finasteride $5 \mathrm{mg}$ and sexual side effects: how many of these are related to a nocebo phenomenon? J Sex Med 2007;4(06):1708-1712

74 Anitha B, Inamadar AC, Ragunatha S. Finasteride-its impact on sexual function and prostate cancer. J Cutan Aesthet Surg 2009;2 (01):12-16

75 Traish AM, Goldstein I, Kim NN. Testosterone and erectile function: from basic research to a new clinical paradigm for managing men with androgen insufficiency and erectile dysfunction. Eur Urol 2007;52(01):54-70

76 Tosti A, Piraccini BM, Soli M. Evaluation of sexual function in subjects taking finasteride for the treatment of androgenetic alopecia. J Eur Acad Dermatol Venereol 2001;15(05):418-421

77 Tosti A, Pazzaglia M, Soli M, et al. Evaluation of sexual function with an international index of erectile function in subjects taking finasteride for androgenetic alopecia. Arch Dermatol 2004;140 (07):857-858

78 Chamsi-Pasha M, Albar MA, Chamsi-Pasha H. Minimizing nocebo effect: Pragmatic approach. Avicenna J Med 2017;7(04):139-143

79 Finn DA, Beadles-Bohling AS, Beckley EH, et al. A new look at the 5alpha-reductase inhibitor finasteride. CNS Drug Rev 2006;12 (01):53-76

80 Dyson TE, Cantrell MA, Lund BC. Lack of association between $5 \alpha-$ Reductase Inhibitors and Depression. J Urol 2020;204(04): 793-798

81 Kim JH, Shim SR, Khandwala Y, Del Giudice F, Sorensen S, Chung BI. Risk of depression after 5 alpha reductase inhibitor medication: meta-analysis. World J Mens Health 2020;38(04):535-544 
82 Schmitz-Dräger BJ, Fischer C, Bismarck E, Dörsam HJ, Lümmen G. [The Prostate Cancer Prevention Trial (PCPT). Relevance for clinical practice]. Urologe A 2007;46(10):1364, 1366-1368, 1370

83 Wallerstedt A, Strom P, Gronberg H, Nordstrom T, Eklund M. Risk of prostate cancer in men treated with $5 \alpha$-reductase inhibitors-A large population-based prospective study. J Natl Cancer Inst 2018;110(11):1216-1221

84 Unger JM, Hershman DL, Till C, et al. Using Medicare claims to examine long-term prostate cancer risk of finasteride in the prostate cancer prevention trial.J Natl Cancer Inst 2018;110(11): 1208-1215

85 Reed AB, Parekh DJ. The utility of 5-alpha reductase inhibitors in the prevention and diagnosis of prostate cancer. Curr Opin Urol 2009;19(03):238-242

86 Carlson RH. PSA test sensitized by finasteride. Oncology Times 2006;28(19):12-13

87 Etzioni RD, Howlader N, Shaw PA, et al. Long-term effects of finasteride on prostate specific antigen levels: results from the prostate cancer prevention trial. J Urol 2005;174(03):877-881

88 D'Amico AV, Roehrborn CG. Effect of $1 \mathrm{mg} /$ day finasteride on concentrations of serum prostate-specific antigen in men with androgenic alopecia: a randomised controlled trial. Lancet Oncol 2007;8(01):21-25

89 Accessed August 29, 2021 at: https://www.cdc.gov/ cliac/docs/addenda/cliac0311/Addendum_D_JAROW.pdf

90 Accessed August 29, 2021 at: https://assets.publishing.service. gov.uk/government/uploads/system/uploads/attachment_data/ file/852392/Finasteride_risk_of_male_breast_cancer.pdf

91 Meijer M, Thygesen LC, Green A, et al. Finasteride treatment and male breast cancer: a register-based cohort study in four Nordic countries. Cancer Med 2018;7(01):254-260

92 Kjærulff TM, Ersbøll AK, Green A, et al. Finasteride Use and risk of male breast cancer: a case-control study using individual-level registry data from Denmark, Finland, and Sweden. Cancer Epidemiol Biomarkers Prev 2019;28(05):980-986

93 Overstreet JW, Fuh VL, Gould J, et al. Chronic treatment with finasteride daily does not affect spermatogenesis or semen production in young men. J Urol 1999;162(04):1295-1300

94 Laborde E, Brannigan RE. Effect of 1-mg dose of finasteride on spermatogenesis and pregnancy. J Androl 2010;31(02): e1-e2

95 Samplaski MK, Lo K, Grober E, Jarvi K. Finasteride use in the male infertility population: effects on semen and hormone parameters. Fertil Steril 2013;100(06):1542-1546

96 Glina S, Neves PA, Saade R, Netto NR Jr, Soares JB, Galuppo AG. Finasteride-associated male infertility. Rev Hosp Clin Fac Med Sao Paulo 2004;59(04):203-205

97 Traish AM. Post-finasteride syndrome: a surmountable challenge for clinicians. Fertil Steril 2020;113(01):21-50
98 Pereira AFJR, Coelho TOA. Post-finasteride syndrome. An Bras Dermatol 2020;95(03):271-277

99 Diviccaro S, Melcangi RC, Giatti S. Post-finasteride syndrome: an emerging clinical problem. Neurobiol Stress 2019;12:100209

100 Rezende HD, Dias MFRG, Trüeb RM. A comment on the postfinasteride syndrome. Int J Trichology 2018;10(06):255-261

101 Trüeb RM, Régnier A, Dutra Rezende H, Gavazzoni Dias MFR. Post-finasteride syndrome: an induced delusional disorder with the potential of a mass psychogenic illness? Skin Appendage Disord 2019;5(05):320-326

102 Chandrashekar BS, Nandhini T, Vasanth V, Sriram R, Navale S. Topical minoxidil fortified with finasteride: an account of maintenance of hair density after replacing oral finasteride. Indian Dermatol Online J 2015;6(01):17-20

103 Sheikh S, Ahmad A, Shoukath AM, et al. A new topical formulation of minoxidil and finasteride improves hair growth in men with androgenetic alopecia. Clin Exp Dermatol Res 2015;6:1

104 Caserini M, Radicioni M, Leuratti C, Annoni O, Palmieri R. A novel finasteride $0.25 \%$ topical solution for androgenetic alopecia: pharmacokinetics and effects on plasma androgen levels in healthy male volunteers. Int J Clin Pharmacol Ther 2014;52 (10):842-849

105 Rai $\mathrm{PB}$, et al. Comparing the therapeutic efficacy of topical minoxidil and finasteride with topical minoxidil and oral finasteride in androgenetic alopecia: a randomized trial. Int J Res Dermatol. 2018;4(03):386-390

106 Marihart S, Harik M, Djavan B. Dutasteride: a review of current data on a novel dual inhibitor of 5alpha reductase. Rev Urol 2005; 7(04):203-210

107 Choi GS, Kim JH, Oh SY, et al. Safety and tolerability of the dual 5alpha reductase inhibitor dutasteride in the treatment of androgenetic alopecia. Ann Dermatol 2016;28(04):444-450

108 Zhou Z, Song S, Gao Z, Wu J, Ma J, Cui Y. The efficacy and safety of dutasteride compared with finasteride in treating men with androgenetic alopecia: a systematic review and meta-analysis. Clin Interv Aging 2019;14:399-406

109 Murugusundram S. Serenoa repens: does it have any role in the management of androgenetic alopecia? J Cutan Aesthet Surg 2009;2(01):31-32

110 Evron E, Juhasz M, Babadjouni A, Mesinkovska NA. Natural hair supplement: friend or foe? Saw Palmetto, a systematic review in alopecia. Skin Appendage Disord 2020;6(06):329-337

111 Guo EL, Katta R. Diet and hair loss: effects of nutrient deficiency and supplement use. Dermatol Pract Concept 2017;7(01):1-10

112 Finner AM. Nutrition and hair: deficiencies and supplements. Dermatol Clin 2013;31(01):167-172

113 Almohanna HM, Ahmed AA, Tsatalis JP, Tosti A. The role of vitamins and minerals in hair loss: a review. Dermatol Ther (Heidelb) 2019;9(01):51-70 